\title{
Membrane-bound Plastid Inclusions in Neoplasia of Nicotiana-Hybrids
}

\author{
G. Gliem and B. Sprey \\ Institut für Pflanzenkrankheiten der Universität Bonn, \\ Institut für Biotechnologie der Kernforschungsanlage \\ Jülich GmbH, F. R. G.
}

Received February 11, 1979

In recent reports ultrastructural changes in neoplasia of Nicotiana as induced after tobacco tumor virus (TTV) infection have been described (Gliem et al. 1976, Gliem and Nienhaus 1978). In this paper the occurrence of inclusions present within the thylakoid membrane during the chloroplast development in both transformed and uninfected cells is compared.

Membrane-bound inclusions have been reported present in healthy tobacco plants or tissue transformed by either Agrobacterium infection or genetical incompatibility (Hohl 1961, Jensen and Valdovinos 1967, Stetler and Laetsch 1969, Boasson et al. 1972, Henry 1975). The occurrence and distribution of these thylakoid inclusions, together with the different terminology used for these inclusions, has been reviewed by Ames and Pivorun (1974). In the following study these structures will be cited as intrathylakoidal inclusions.

\section{Material and methods}

Nicotiana clevelandii $\times$ Nicotiana glutinosa plants were cultivated for 5-6 weeks under greenhouse conditions. For tumor induction plants were inoculated by needle pricking with sap from freshly harvested tumors. Neoplasia appeared about 2 weeks after inoculation. Samples from 2 to 20 days old tumors and controls from healthy plants were collected and used for electron microscopy.

Pieces of leaves and tumors $\left(1 \mathrm{~mm}^{2}\right)$ were fixed in $0.1 \mathrm{M}$ phosphate-buffered $2.5 \%$ glutaraldehyde, $\mathrm{pH} 7.2$, rinsed in phosphate buffer and postfixed in $2 \% \mathrm{OsO}_{4}$. After dehydration in an acetone series, samples were embedded in low viscosity epoxide resin (Spurr 1969). Thin sections (Ultrotome III, LKB) were poststained with lead citrate for 3 minutes in the case of healthy plant material and up to 20 minutes, when tumor tissue was used (Venable and Coggeshall 1965). Samples were examined in an Elmiskop I (Fa. Siemens).

\section{Results}

Early proplastid stages in tumor cells from plants 16 days after infection show partially swollen thylakoids. The membranes exhibited vesicle-like lamellae (Fig. 1). In later stages of proplastid development an increased amount of dilated inner plastid membranes were found, showing a translucent contents with some 

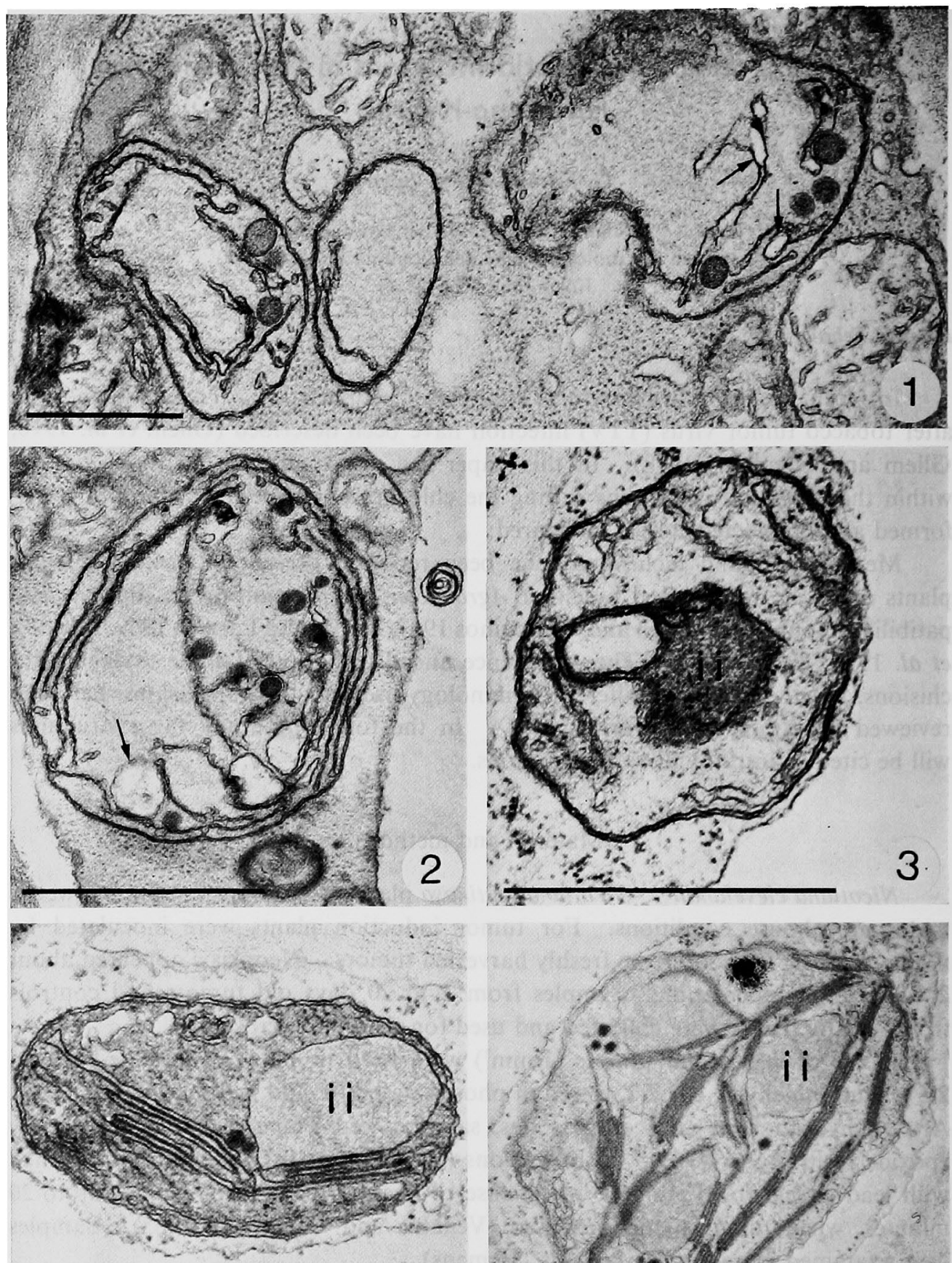

4

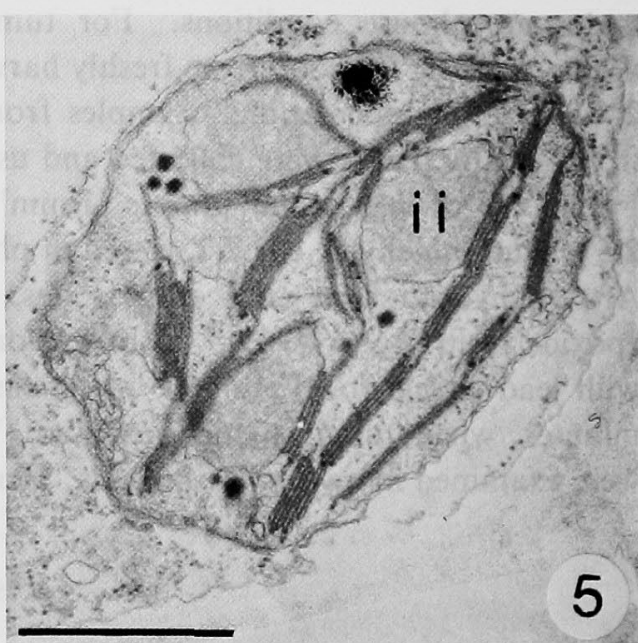

Figs. 1-5. Different stages of plastid development in Nicotiana neoplasia as induced by tobacco tumor virus. 1-3, show proplastids with primary lamellae and vesicle-like membranes. Dilated membranes marked by arrows possibly represent first steps of thylakoid dilations (Figs. 2, 3). 4-5, the contents of intrathylakoidal inclusions (i.i.) in young chloroplasts seems to be composed of condensed granular material (Figs. 4, 5). The bar length in all micrographs represents $1 \mu \mathrm{m}$. 

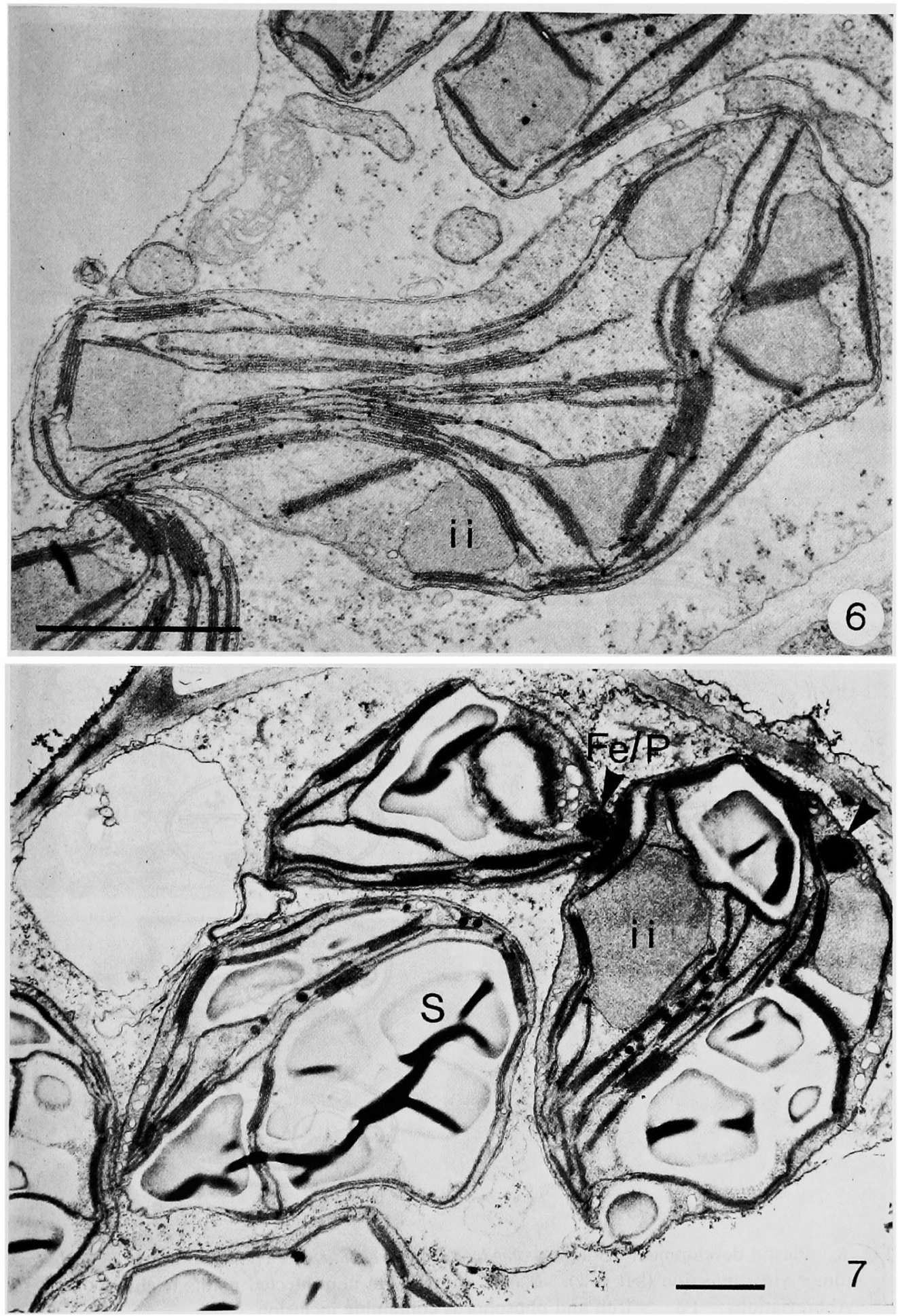

Fig. 6. Intrathylakoidal inclusions (i.i.) in plastids of an 8 day-old tumor cell with poorly developed grana stacks.

Fig. 7. Plastids with intrathylakoidal inclusions (i.i.) in cells of a 12 day-old tumor. They are characterized by an increased amount of starch (S) and iron-phosphorus containing inclusions $(\mathrm{Fe} / \mathrm{P})$. 


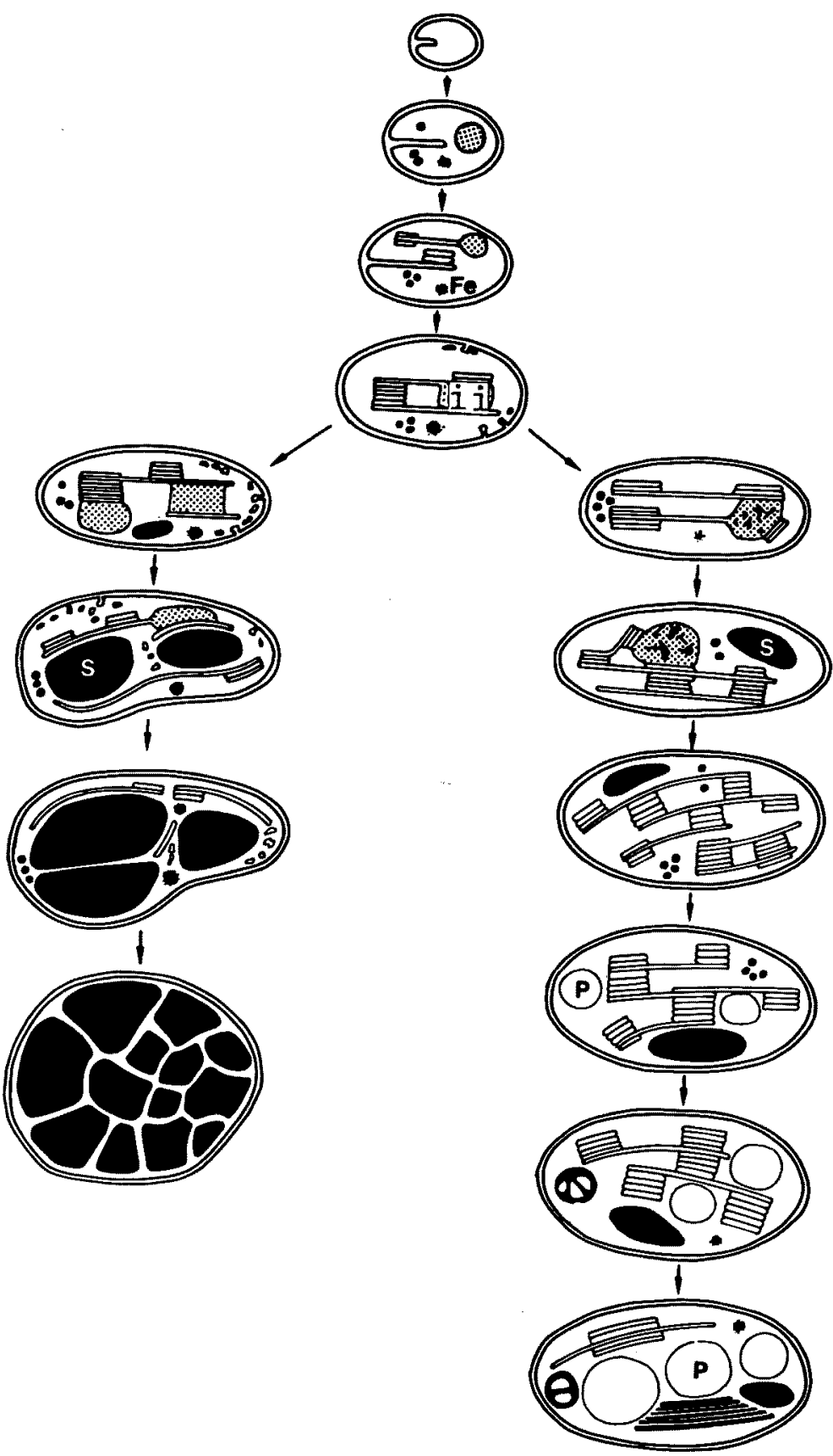

Fig. 8. Plastid development in tumors of Nicotiana clevelandii $\times N$. glutinosa induced by tobacco tumor virus infection (left row). Plastid ontogeny in non-infected plants (right row).

Abbreviations: $\mathrm{Fe}=$ iron and phosphorus containing inclusion

i.i. = intrathylakoidal inclusion

$\mathbf{P}=$ plastoglobule, $\mathbf{S}=$ starch 

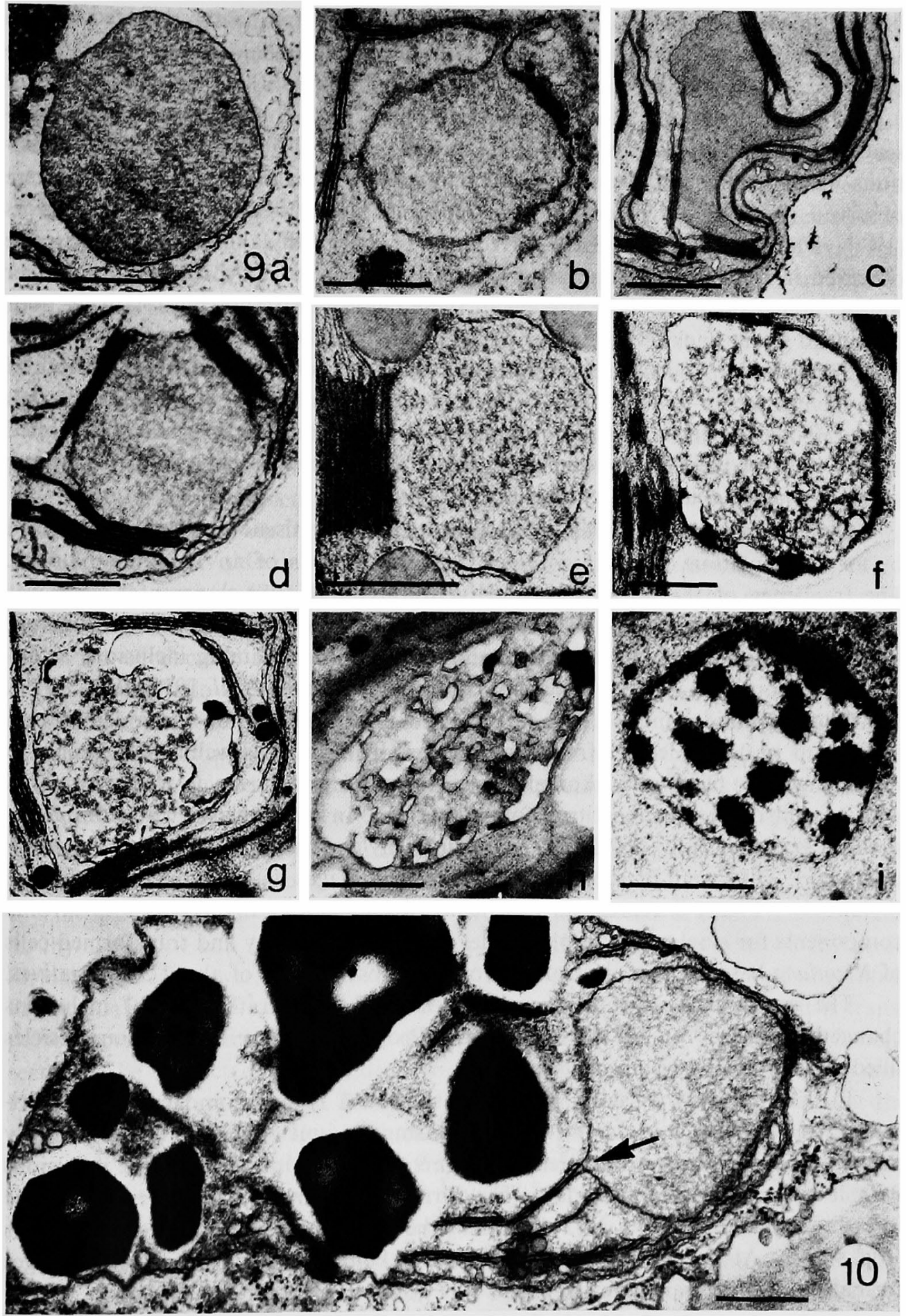

Figs. 9. Variability in the appearance of the contents of intrathylakoidal inclusions as present in different stages of plastid development. For details see text.

Fig. 10. Membrane of an intrathylakoidal inclusion in connection with a thylakoid. 
fibrillar material (Fig. 2). These dilations were less frequently detected in uninfected controls. With further plastid development thylakoids showing a contents with an enhanced electron density were noticed (Fig. 3).

Younger stages of chloroplast development are characterized by the presence of stroma lamellae and a poorly expressed grana formation. Thylakoid dilations with a more homogeneous contents and an increased electron density were observed (Fig. 4). Inclusions of this appearence prevailed in 5 day-old tumors (19 days after infection). Highest frequencies of intrathylakoidal inclusions were observed in the subsequent stage of plastid development. Up to 5 inclusions per thin-sectioned organelle were present in these chloroplasts showing relatively poor developed grana stacks (Fig. 5). In older tumors (age 8 days) no significant changes in the distribution and ultrastructure of the intrathylakoidal inclusions were noticed (Fig. 6). It seems that no further increase in the formation of thylakoids takes place. Thus, the grana formation of plastids in tumor tissue appears to be inhibited at this stage of plastid development. This is in contrast to healthy plants, where grana formation continued (cf. Fig. 8).

Older stages of chloroplast development in tumor tissue can be characterized by an accumulation of starch. Figure 7 shows plastids of an 12 day-old tumor. The frequency of the intrathylakoidal inclusions in this developmental stage was found to be reduced. Electron dense structures observed in the stroma of different stages of plastid development are iron and phosphorus containing inclusions as analysed previously by X-ray microanalysis techniques (Sprey et al. 1976, 1978, 1979).

Intrathylakoidal inclusions were detected in plastids of the postapical region and in up to $0.5 \mathrm{~cm}$ leaflets from uninfected controls as well. Differences in the arrangement of intrathylakoidal inclusions were found, however, when compared with plastid development in transformed tissue. In chloroplasts with completely stacked grana of fully developed leaves intrathylakoidal inclusions were scarcely observed. They were present in senescent plastid stages with a reduced amount of thylakoids. Figure 8 represents a scheme of changes in thylakoids and stroma components for single steps of plastid development in healthy and transformed cells of Nicotiana based on the electron microscopic observations of about 500 negatives.

The appearance of the internal material of the intrathylakoidal inclusions changed depending on the plastid stages. Proplastids can either contain vesicles filled with or devoid of any material.

In TTV-infected cells the inclusions showed a rather homogeneously distributed contents (Fig. $9 \mathrm{a}-\mathrm{c}$ ). With an increasing amount of thylakoids less densely packed inner material was noticed. Figure $9 \mathrm{~d}-\mathrm{f}$ shows intrathylakoidal inclusions with loosely aggregated material. The formation of dense aggregates within the intrathylakoidal inclusions was followed by the appearance of electron dense clusters (Fig. $9 \mathrm{f}-\mathrm{i}$ ). At the periphery of the intrathylakoidal inclusions from older stages formation of membrane-like structures was observed (Fig. $9 \mathrm{f}-\mathrm{h}$ ).

\section{Discussion}

The possible function of intrathylakoidal inclusions occurring in plastids of 
different species of higher plants (cf. Casadoro and Rascio 1978) is still a matter of speculation. Hypotheses based on developmental studies of chloroplasts concern their possible storage function (Marinos 1967, Newcomb 1967, Salema and Badenhuizen 1967) or their role in thylakoid formation processes (Israel and Steward 1967, Blackwell et al. 1969, Stetler and Laetsch 1969, Boasson et al. 1972, Ames and Pivorun 1974, Cran and Possingham 1974). Thus the construction of the thylakoid system in these species is believed to be correlated with a decrease of intrathylakoidal inclusions (Boasson et al. 1972, Ames and Pivorun 1974). This finding can be confirmed by our results on chloroplast development in uninfected hybrids of Nicotiana clevelandii $\times N$. glutinosa. Until now there is no experimental approach to correlate the polypeptide pattern of isolated thylakoids of young plastids from tobacco containing intrathylakoidal inclusions with thylakoidal fractions from mature chloroplasts with a reduced amount of intrathylakoidal inclusions. Experiments based on the comparison of membrane lipids and polypeptides from both chloroplast types are in progress in this laboratory.

It has been presumed that the internal material of the intrathylakoidal inclusions may be used up for the construction of the thylakoids (Israel and Steward 1967, Jensen and Valdovinos 1967, Stetler and Laetsch 1969, Boasson et al. 1972). Evidence to support this theory is given by the decrease of the material within the intrathylakoidal inclusions during formation of grana lamellae when followed by means of electron microscopy. The changes and the decrease of the contents of the intrathylakoidal inclusions from plastids of different development stages give structural support to this hypothesis (cf. Fig. 9). Experiments after tryptic digestion of the intrathylakoidal inclusions revealed their proteinaceous nature (Ames and Pivorun 1974). Using diaminobenzidine as a cytochemical marker Jensen (1974) supposes the existence of peroxidases within the intrathylakoidal inclusions.

Besides the gap of information about the function of these inclusions, their mode of formation in proplastids and young chloroplasts is unknown. As intrathylakoidal inclusions were observed in proplastids devoid of what is interpreted as thylakoids, it can be argued that their formation is independent of thylakoid formation. Moreover, it is unknown to what extent the content of the intrathylakoidal inclusions can be synthesized in the plastid stroma and how it is transported across the lamellae. Since the presence of the intrathylakoidal inclusions seems to be restricted to plastids of only some higher plant species (Ames and Pivorun 1974, Casadora and Rascio 1978) care should be taken generalizing the existence of the intrathylakoidal inclusions as a prerequisite for plastid development. It is recognized that the generally accepted mode of plastid development (Mühlethaler and Frey-Wyssling 1959, Gunning and Jagoe 1967) is valid without the existence of the intrathylakoidal inclusions.

Chloroplasts from tumor tissue showed an increase in the number of intrathylakoidal inclusions when compared with uninfected plants. It is likely that this phenomenon is due to a possible blockage of plastid development after transformation. As chloroplasts in infected tissue never reached stages with fully developed grana thylakoids (Gliem and Nienhaus 1978) it is assumed that the inhibition of a normal thylakoid development is indicated by the increased amount of 
intrathylakoidal inclusions.

$\mathrm{X}$-ray microanalysis of iron and phosphorus containing stroma inclusions (Sprey et al. 1976, 1977, 1978) from infected and healthy plants gives additional evidence for the idea of an inhibited chloroplast development caused by transformation. In chloroplasts from tumor tissue atomic ratios and absolute weight percentages of both elements were found to be reduced (Sprey et al. 1976). The composition of the intrathylakoidal inclusions in plastids from infected plants corresponds to those present in young chloroplasts of uninfected controls. Therefore evidence is given that the infection inhibits normal chloroplast development resulting in chloroplasts with a poorly expressed grana system and an increased amount of intrathylakoidal inclusions. It is assumed that this phenomenon of an inhibited plastid development is not directly caused by the virus infection but is evoked by cell neoplasia.

\section{Acknowledgements}

This work has been supported in part (B.S.) by the DFG (Sonderforschungsbereich 160). The skilled assistance of Mrs. G. Król is very much appreciated.

\section{Summary}

The development of plastids in tumor cells of Nicotiana-hybrids induced by the tobacco tumor virus (TTV) was studied by means of transmission electron microscopy and compared to uninfected controls. Proplastids and young chloroplasts in tumor cells contain intrathylakoidal inclusions. In contrast to controls the plastid development is delayed in young chloroplast stages. These are characterized by a poorly developed grana system and the formation of large starch aggregations. The ultrastructural changes in the intrathylakoidal inclusions were studied at the different stages of plastid development. Their possible functional role during plastid ontogeny is discussed.

Key Words: chloroplast development, ultrastructure, intrathylakoidal inclusions, tobacco tumor virus, neoplasia, Nicotiana

\section{References}

Ames, J. H., and Pivorum, J. P. 1974. A cytochemical investigation of a chloroplast inclusion. Amer. Bot. J. 61 (7): 794-797.

Blackwell, S. T., Laetsch, W. M. and Hyde, B. B. 1969. Development of chloroplast fine structure in Aspen tissue culture. Amer. J. Bot. 56 (4): 457-463.

Boasson, R., Laetsch, W. M. and Price, I. 1972. The etioplast-chloroplast transformation in tobacco: Correlation of ultrastructure, replication and chlorophyll synthesis. Amer. J. Bot 59 (3): 217-223.

Casarodo, G. and Rascio, N. 1978. Thylakoid membranes in sunflower and other plants. J. Ultrastruct. Res. $65: 30-35$.

Cran, D. G. and Possingham, J. V. 1974. The effect of cell age on chloroplast structure and chlorophyll in cultured spinach leaf discs. Protoplasma 79: 197-213. 
Gliem, G., Sprey, B. und Nienhaus, F. 1976. Elektronenmikroskopische Untersuchungen cytologischer Veränderungen in Tabakpfianzen durch ein neues Tabak-Tumorvirus. Proceedings of Internat. Symp. on Crop Protection, Med. Fac. Landbouw. Rijksuniv. Gent. 41/2: 771-779.

- und Nienhaus, F. 1978. Plastidenentwicklung in Tabakhybriden nach Infektion mit dem TabakTumorvirus und nach Agrobacterium tumefaciens Infektion. Z. Pflanzenkrankh. u. Pflschutz 85 (3/4): 247-253.

Gunning, B. E. S. and Jagoe, M. P. 1967. The prolamellar body. In: Biochemistry of Chloroplasts. Goodwin, T. W., editor, Academic Press Inc. New York 2: 655-676.

Henry, E. W. 1975. Peroxidases in tobacco abscission zone tissue III. Ultrastructural localisasation in thylakoids and membrane-bound bodies of chloroplasts. J. Ultrastruct. Res. 52: 289-299.

Hohl, H. 1961. Über die submikroskopische Struktur hyperplastischer Gewebe von Datura stramonium L. Phytopath. Z. 40: 317-356.

Israel, H. W. and Steward, F. C. 1967. The fine structure and development of plastids in cultured cells of Daucus carota. Ann. Bot. 31:1-18.

Jensen, T. E. and Valdovinos, J. G. 1967. Fine structure of abscission zones I. Abscission zones of the pedicells of tobacco and tomato flowers at anthesis. Planta 77: 298-318.

Marinos, N. G. 1967. Multifunctional plastids in the meristematic region of potato tuber buds. J. Uitrastruct. Res. 17: 91-113.

Mühlethaler, K. und Frey-Wyssling, A. 1959. Entwicklung und Struktur der Plastiden. J. Biophys. Biochem. Cytol. 6: 507-512.

Newcomb, E. H. 1967. Fine structure of protein storing plastids in bean root tips. J. Cell Biol. 33: 143-163.

Salema, R. and Badenhuizen, N. P. 1967. The production of reserve starch granules in the amyloplasts of Pelliona daveauana NE. Br. J. Ultrastruct. Res. 20: 389-399.

Sprey, B., Gliem, G. and Jánossy, A. G. S. 1976. Iron containing inclusions in chloroplasts of Nicotiana clevelandii $\times$ Nicotiana glutinosa $\mathrm{I}$. X-ray microanalysis and ultrastructure. $\mathbf{Z}$. Pflanzenphysiol. 79: 165-176.

- - - and - 1977. Changes in the iron and phosphorus content of stroma inclusions during etioplast-chloroplast development in Nicotiana. Z. Naturforsch. 32 (c): 158-159.

,-- and - 1978. Iron and phosphorus containing inclusions in chloroplasts of Nicotiana clevelandii $\times N$. glutinosa II. Development of etioplast to chloroplasts in cotyledons. Z. Pflanzenphysiol. 88: 69-82.

- and Gliem, G. 1979. Iron and phosphorus containing inclusions in proplastids of Nicotiana. Cytologia 44: 581-584.

Spurr, A. R. 1969. A low viscosity epoxy resin embedding medium for electron microscopy. J. Ultrastruct. Res. 26: 31-43.

Stetler, D. A. and Laetsch, W. U. 1969. Chloroplast development in Nicotiana tabacum "Maryland mammoth". Amer. J. Bot. 56: 260-270.

Venable, J. J. and Coggeshall, R. 1965. A simplified lead citrate stain for use in electron microscopy. J. Cell. Biol. 25: 407-408. 